\title{
artigo
}

Aquino, L.M.; Magalhães, R.E.C.C.; Kawakami, R.M.S.A.; Romão, S.R.M.; Cardoso da Silva, S.; Santos, V.F.C.;

Práticas interprofissionais em saúde e enfermagem na unidade de pronto atendimento: revisão integrativa

\section{Práticas interprofissionais em saúde e enfermagem na unidade de pronto atendimento: revisão integrativa}

\author{
Interprofessional practices in health na nursing in the emergency care unit: intergrative review \\ Prácticas interproesionales em salud Y ENFERMERÍA EM LA UNIDAD DE urgencias: revisíon integrativa
}

\begin{abstract}
RESUMO
Objetivo: Identificar as práticas interprofissionais em saúde e enfermagem que podem ser desenvolvidas na unidade de pronto atendimento, evidenciadas na literatura. Método: Foi realizada uma pesquisa de revisão integrativa, limitada em artigos de 2015 a 2020, na biblioteca eletrônica do Centro Latino-Americano e do Caribe de Informação em Ciências da Saúde (BIREME), na base de dados Literatura Latino-Americana e do Caribe em Ciências da Saúde (LILACS), na Scientific Electronic Library Online (SciELO) e Medical Literature Analysis and Retrieval Sistem on-line (MEDLINE), utilizando os descritores "relações interprofissionais" "comportamento cooperativo" "equipe de assistência ao paciente" "enfermagem" e "emergências". Sendo feito a pesquisa isolada e o cruzamento entre eles. A análise de dados e discussão foram baseadas na revisão de literatura. Resultados: A busca resultou em 143 artigos, sendo selecionados 10 artigos que atenderam os critérios de inclusão. Conclusão: 0 trabalho interprofissional em saúde e enfermagem permite satisfação do usuário, do profissional e uma assistência de qualidade.
\end{abstract}

DESCRITORES: Relações interprofissionais; Comportamento cooperativo; Equipe de assistência ao paciente; Enfermagem; Emergências.

\begin{abstract}
Objective: To identify the interprofessional practices in health and nursing that can be developed in the emergency care unit, as evidenced in the literature. Method: An integrative review research was conducted, limited in articles from 2015 to 2020, in the electronic library of the Latin American and Caribbean Center for Health Sciences Information (BIREME), in the Latin American and Caribbean Literature in Sciences database da Saúde (LILACS), Scientific Electronic Library Online (SciELO) and Medical Literature Analysis and Retrieval Sistem online (MEDLINE), using the descriptors "interprofessional relations" "cooperative behavior" "patient care team" "nursing" and "Emergencies". Being done the isolated research and the crossing between them. Data analysis and discussion were based on the literature review. Results: 143 articles were found, of which 10 articles that met the inclusion criteria were selected. Conclusion: The interprofessional work in health and nursing allows user, professional satisfaction and quality care. DESCRIPTORS: Interprofessional relations; Cooperative behavior; Patient care team; Nursing; Emergencies.
\end{abstract}

\section{RESUMEN}

Objectivo: Identificar las prácticas interprofesionales en salud y enfermería que se pueden desarrollar en la unidad de urgencias, como se evidencia en la literatura. Método: Se realizó una investigación de revisión integradora, limitada en artículos de 2015 a 2020, en la biblioteca electrónica del Centro Latinoamericano y del Caribe de Información en Ciencias de la Salud (BIREME), en la base de datos de Literatura en Ciencias de América Latina y el Caribe da Saúde (LILACS), Scientific Electronic Library Online (SciELO) y Medical Literature Analysis and Retrieval Sistem online (MEDLINE), utilizando los descriptores "relaciones interprofesionales" "comportamiento cooperativo" "equipo de atención al paciente" "enfermería" y "Emergencias". Realizándose la investigación aislada y el cruce entre ellos. El análisis y la discusión de los datos se basaron en la revisión de la literatura. Resultados: se encontraron 143 artículos, de los cuales se seleccionaron 10 artículos que cumplieron com los criterios de inclusión. Conclusión: El trabajo interprofesional en salud y enfermería permite la satisfacción del usuario, profesional y atención de calidad.

DESCRIPTORES: Relaciones interprofesionales; Comportamiento cooperativo; Equipo de atención al paciente; Enfermería; Emergencias.

RECEBIDO EM: 28/08/2020 APROVADO EM: 17/09/2020

\section{Luciene Marques de Aquino}

Acadêmica de enfermagem do Centro Universitário de Várzea Grande (UNIVAG).

ORCID: 0000-0003-3560-8094 


\section{Ruth Eugênia de Campos Curvo Magalhães}

Acadêmica de enfermagem do Centro Universitário de Várzea Grande (UNIVAG).

ORCID: 0000-0001-5192-1924

\section{Roselma Marcele da Silva Alexandre Kawakami}

Enfermeira. Especialista em Auditoria dos Seviços de Saúde. Especialista em Vigilância em Saúde. Mestra do Pograma de Pós-Graduação em Enfermagem da Universidade Federal de Mato Grosso. Atualmente docente no Centro Universitário-UNIVAG. ORCID: 0000-0001-5581-8115

\section{Samira Rodrigues Mendes Romão}

Acadêmica de enfermagem do Centro Universitário de Várzea Grande (UNIVAG). ORCID: 0000-0003-4911-2321

\section{Simone Cardoso da Silva}

Acadêmica de enfermagem do Centro Universitário de Várzea Grande (UNIVAG). ORCID: 0000-0002-1036-2879

\section{Valquiria Fabiana Correa Santos}

Acadêmica de enfermagem do Centro Universitário de Várzea Grande (UNIVAG). ORCID: 0000-0002-4894-6554

\section{INTRODUÇÃO}

D e acordo com o Ministério da Saú$\mathrm{de}^{1}$, a Unidade de Pronto Atendimento (UPA) funciona 24 horas, presta atendimento resolutivo, qualificado e integrado aos pacientes em diferentes condições de saúde. Visa atender as urgências e emergências nos processos agudos das doenças crônicas, pequenas cirurgias, traumas, investigação diagnostica inicial, entre outros. A partir da realidade de saúde os profissionais da UPA deverão garantir através do fluxo da rede de atenção à saúde a continuidade do tratamento encaminhando o indivíduo para outros pontos de atenção à saúde.

$\mathrm{Na}$ prática do processo de trabalho em saúde, as atividades são realizadas por equipes multiprofissionais que atendem o paciente de forma individual e centrada na sua necessidade ${ }^{2}$. Nessa perspectiva o trabalho em equipe é importante, uma vez que, possibilita interação entre as pessoas, permitindo colaboração dos profissionais na realização de cuidados ${ }^{4}$. Nesse contexto, adotar a prática interprofissional como estratégia de trabalho e formação com intuito de promover uma assistência integral ao usuário é o caminho a ser trilhado no serviço de saúde ${ }^{3}$.

Desta forma, considera-se interprofissionalidade o momento em que diferentes profissões convivem e se relacionam em um mesmo espaço e compartilham através da comunicação as decisões para melhor produção do cuidado em saúde 5 . A atuação interprofissional se torna um processo ativo, onde os profissionais aprendem a trabalhar juntos, reconhecer o trabalho do outro, definir um objetivo da equipe, realizar de forma compartilhada projetos e técnicas para atender o usuário em situações complexas ${ }^{6}$.

A literatura aponta que os estudos realizados sobre interprofissionalidade exploram conteúdos sobre educação interprofissional em saúde ${ }^{7}$ e prática interprofissional ${ }^{8}$. Estudo também aborda sobre a colaboração interprofissional e o trabalho em equipe4. Além disso, existe estudo sobre interprofissionalidade na atenção básica ${ }^{9}$, e poucos estudos encontrados sobre interprofissionalidade nas unidades de urgência e emergência ${ }^{10,11}$.

Neste sentido, no Brasil a educação interprofissional vem se destacando, visto que esta forma de se trabalhar o ensino, repercute em melhoria no atendimento do Sistema Único de Saude (SUS) contribuindo para a formação de profissionais da saúde capaci$\operatorname{tados}^{12}$. Por essa razão, é necessário estudos sobre a prática interprofissional em saúde e enfermagem na UPA para que os profissionais tenham sua prática baseada em evidência. Neste contexto, entende-se que existe uma lacuna no sentido buscar evidencias sobre essa prática para que seja desenvolvida nos serviços de saúde.

Diante deste contexto surgiu a seguinte questão norteadora: quais são as práticas interprofissionais em saúde e enfermagem que podem ser desenvolvidas na Unidade de Pronto Atendimento? Por essa razão, o objetivo do estudo é de identificar as práticas interprofissionais em saúde e enfermagem que podem ser desenvolvidas na unidade de pronto atendimento evidenciadas na literatura.

\section{MÉTODO}

Trata-se de uma revisão integrativa da literatura que consiste nas etapas de identificação do tema, elaboração da questão norteadora, busca de estudos experimentais ou não experimentais, coleta de dados, análise crítica dos estudos selecionados, interpretação dos resultados e apresentação dos dados da revisão integrativa ${ }^{13}$. A coleta foi feita no mês de junho de 2020 pelas 5 acadêmicas, considerando o limite temporal entre 2015 a 2020. Foi consultada a biblioteca eletrônica do Centro Latino-Americano e do Caribe de Informação em Ciências da Saúde (BIREME), as bases de dados Literatura Latino-Americana e do Caribe em Ciências da Saúde (LILACS), Scientific Electronic Library Online 


\section{artigo}

Aquino, L.M.; Magalhães, R.E.C.C.; Kawakami, R.M.S.A.; Romão, S.R.M.; Cardoso da Silva, S.; Santos, V.F.C.

Práticas interprofissionais em saúde e enfermagem na unidade de pronto atendimento: revisão integrativa

(SciELO) e Medical Literature Analysis and Retrieval Sistem on-line (MEDLI$\mathrm{NE}$ ). Os descritores utilizados: relações interprofissionais, comportamento cooperativo, equipe de assistência ao paciente, enfermagem e emergências. Foi utilizado o operador boleano AND para o cruzamento dos descritores, tendo em vista a pouca quantidade de artigos foi necessário desenvolver busca isolada de descritores.

Os critérios de inclusão foram: artigos científicos dos últimos cinco anos (2015-2020), textos completos, disponíveis online, no idioma português e que contemplam o objetivo do estudo. Foram excluídos artigos de revisão de literatura e os repetidos. O quadro 1 representa as estratégias de busca:

\section{Figura 1. Fluxograma para Seleção de artigos. Várzea Grande, MT, Brasil, 2020}

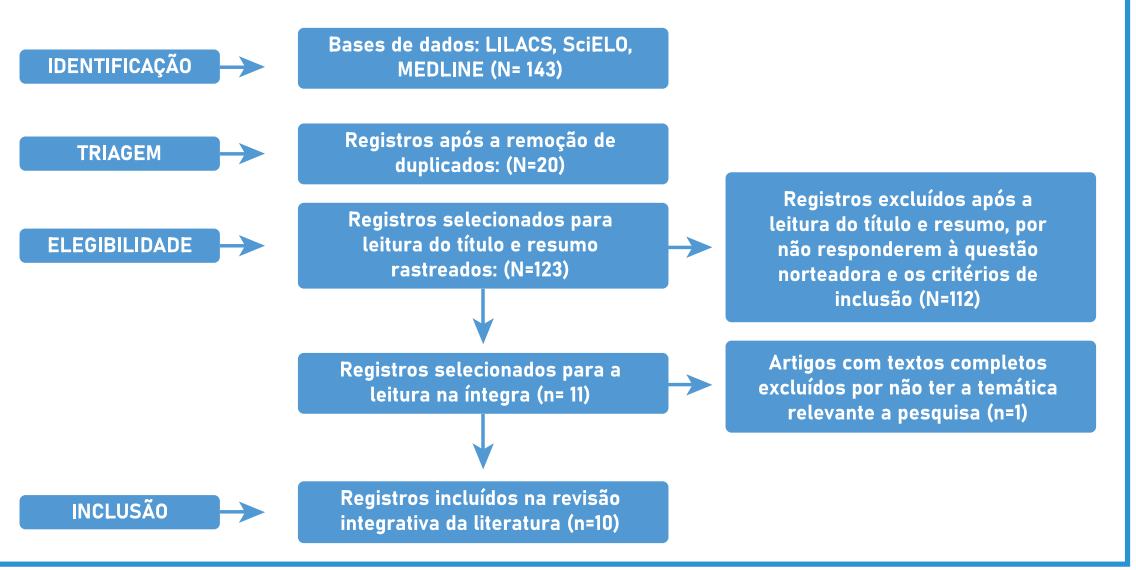

Para a seleção dos estudos, foram adotadas as recomendações do Preferred Reporting Items for Systematic Reviews and Meta-Analyses (PRISMA), conforme apresentado na Figura 1.

Os dados foram coletados de forma sistemática, por meio de um instrumento validado por Ursi, identificando o texto, instituição, tipo de publicação, características metodológicas e avaliação do rigor metodológico. Após a leitura dos artigos foi realizada a análise e interpretação, extraindo as informações mais significativas dos resultados. A discussão dos resultados obtidos foi a luz da revisão de literatura do projeto de pesquisa e respeitou a lei sobre os direitos autorais de $\mathrm{n}^{\circ} 9.610$ de 19 de fevereiro de 1998.

\section{RESULTADOS}

Esta revisão integrativa da literatura foi construída a partir da análise de 10 artigos. No quadro a seguir, são apresentadas as características destes:

Quadro 1: Estratégias de busca de artigos científicos, 2015-2020, pesquisados em junho de 2020:

\begin{tabular}{|l|c|c|c|}
\hline $\begin{array}{l}\text { CRUZAMENTO DOS DESCRITORES UTILIZANDO OPERADOR } \\
\text { BOLEANO AND }\end{array}$ & $\begin{array}{c}\text { RESULTADOS } \\
\text { ENCONTRADOS }\end{array}$ & REPETIDOS & $\begin{array}{c}\text { ARTIGOS } \\
\text { SELECIONADOS }\end{array}$ \\
\hline Relações interprofissionais and enfermagem & 14 & 0 & 2 \\
\hline Comportamento cooperativo and relações interprofissionais & 12 & 2 & 3 \\
\hline Relações interprofissionais and equipe de assistência ao paciente & 16 & 1 & 2 \\
\hline Comportamento cooperativo and emergência & 12 & 4 & 1 \\
\hline Comportamento cooperativo and enfermagem & 12 & 3 & 2 \\
\hline Descritor isolado & Resultados & Repetidos & Artigos \\
\hline Relações interprofissionais & 46 & 6 & 0 \\
\hline Comportamento cooperativo & 31 & 4 & 0 \\
\hline TOTAL & 143 & 20 & 10 \\
\hline
\end{tabular}

Quadro 2: Artigos levantados nas bases de dados LILACS, SciELO E MEDLINE, 2015-2020.

\begin{tabular}{|c|c|c|c|c|c|}
\hline $\mathrm{N}^{\circ}$ & BASE DE DADOS & TÍTULO & AUTOR, ANO & OBJETIVO & TIPO DE ESTUDO \\
\hline 1 & LILACS & $\begin{array}{l}\text { O exercício da inter- } \\
\text { profissionalidade e } \\
\text { da intersetorialidade } \\
\text { como arte de cuidar: } \\
\text { inovações e desafios. }\end{array}$ & $\begin{array}{l}\text { Reuter CLO, } \\
\text { Santos VCF, } \\
\text { Ramos AR } \\
2018 .\end{array}$ & $\begin{array}{c}\text { Analisar as inovações e os desafios } \\
\text { apontados pelas coordenadoras da } \\
\text { atenção básica para o exercício interpro- } \\
\text { fissional e intersetorial, e a importância } \\
\text { dessas práticas como potencializado- } \\
\text { ras do cuidar e do ensinar a cuidar em } \\
\text { Enfermagem, no âmbito da Atenção } \\
\text { Primária em Saúde. }\end{array}$ & $\begin{array}{l}\text { Exploratório com } \\
\text { abordagem qualita- } \\
\text { tiva }\end{array}$ \\
\hline
\end{tabular}




\begin{tabular}{|c|c|c|c|c|c|}
\hline 2 & LILACS & $\begin{array}{c}\text { Interdisciplinaridade e } \\
\text { interprofissionalidade } \\
\text { na estratégia saúde } \\
\text { da familia. }\end{array}$ & $\begin{array}{c}\text { Farias DN, } \\
\text { Ribeiro KSQS, } \\
\text { Anjos UU, Brito } \\
\text { GEG. } 2018 .\end{array}$ & $\begin{array}{c}\text { Caracterizar a perspectiva } \\
\text { interdisciplinar na Estratégia Saúde } \\
\text { da Familia numa capital do Brasil, } \\
\text { relacionando-a à interprofissionalidade. }\end{array}$ & $\begin{array}{c}\text { Transversal } \\
\text { exploratório com } \\
\text { abordagem qualitativa } \\
\text { e quantitativa. }\end{array}$ \\
\hline 3 & LILACS & $\begin{array}{l}\text { Prática colaborativa: } \\
\text { potencialidades e } \\
\text { desafios para o en- } \\
\text { fermeiro no contexto } \\
\text { hospitalar. }\end{array}$ & $\begin{array}{l}\text { Caram CS, } \\
\text { Rezende LC, } \\
\text { Brito MJM. M. } \\
2017 .\end{array}$ & $\begin{array}{l}\text { Compreender a configuração da prática } \\
\text { colaborativa no contexto hospitalar, na } \\
\text { perspectiva do enfermeiro. }\end{array}$ & $\begin{array}{l}\text { Estudo de caso com } \\
\text { abordagem qualita- } \\
\text { tiva. }\end{array}$ \\
\hline 4 & MEDLINE & $\begin{array}{l}\text { Práticas interprofis- } \\
\text { sional colaborativa no } \\
\text { serviço de emer- } \\
\text { gência: atribuições } \\
\text { privativas e compar- } \\
\text { tilhadas dos fisiotera- } \\
\text { peutas. }\end{array}$ & $\begin{array}{l}\text { Batista REA, } \\
\text { Peduzzi M. } \\
2018 .\end{array}$ & $\begin{array}{c}\text { Mapear as atribuições específicas e } \\
\text { compartilhadas dos fisioterapeutas } \\
\text { que atuam em equipes de serviços de } \\
\text { emergência. }\end{array}$ & $\begin{array}{l}\text { Transversal explora- } \\
\text { tório com abordagem } \\
\text { quantitativa. }\end{array}$ \\
\hline 5 & LILACS & $\begin{array}{l}\text { Relacionamento } \\
\text { interprofissional: } \\
\text { identificação de } \\
\text { comportamento para } \\
\text { trabalho em equipe } \\
\text { em unidade corona- } \\
\text { riana. }\end{array}$ & $\begin{array}{l}\text { Goulart BF, } \\
\text { Parreira BDM, } \\
\text { Noce LGA, } \\
\text { Henriques SH, } \\
\text { Simões ALA, } \\
\text { Chaves LDP. } \\
2019 .\end{array}$ & $\begin{array}{l}\text { Identificar, na equipe multiprofissional } \\
\text { da unidade coronariana, comportamen- } \\
\text { tos facilitadores e dificultadores, para o } \\
\text { trabalho em equipe, na perspectiva do } \\
\text { relacionamento interprofissional. }\end{array}$ & $\begin{array}{c}\text { Descritivo com abor- } \\
\text { dagem qualitativa. }\end{array}$ \\
\hline 6 & SciELO & $\begin{array}{l}\text { Colaboração in- } \\
\text { terprofissional na } \\
\text { estratégia saúde da } \\
\text { familia: implicações } \\
\text { para a produção do } \\
\text { cuidado e a gestão do } \\
\text { trabalho. }\end{array}$ & $\begin{array}{c}\text { Matuda CG, } \\
\text { Pinto NRS, } \\
\text { Martins CL, } \\
\text { Frazão P. } 2015 .\end{array}$ & $\begin{array}{l}\text { Captar a percepção de profissionais } \\
\text { que atuam na atenção primária à saúde } \\
\text { sobre o trabalho compartilhado e a } \\
\text { colaboração interprofissional. }\end{array}$ & $\begin{array}{c}\text { Descritivo explorató- } \\
\text { rio com abordagem } \\
\text { qualitativa. }\end{array}$ \\
\hline 7 & MEDLINE & $\begin{array}{l}\text { Prática colaborativa } \\
\text { interprofissional e } \\
\text { assistência em enfer- } \\
\text { magem. }\end{array}$ & $\begin{array}{l}\text { Santos GLA, } \\
\text { Valadares GV, } \\
\text { Santos SS, } \\
\text { Moraes CRBM, } \\
\text { Mello JCM, Vi- } \\
\text { dal LLS. } 2020 .\end{array}$ & $\begin{array}{l}\text { Refletir sobre os argumentos que } \\
\text { ampliam a compreensão da atuação } \\
\text { do enfermeiro, considerando a prătica } \\
\text { colaborativa e buscando possíveis nexos } \\
\text { entre os elementos substantivos da } \\
\text { assistência em enfermagem e a prática } \\
\text { colaborativa interprofissional. }\end{array}$ & $\begin{array}{c}\text { Teórico reflexivo com } \\
\text { abordagem qualita- } \\
\text { tiva. }\end{array}$ \\
\hline 8 & LILACS & $\begin{array}{l}\text { Retratos da prática } \\
\text { interprofissional cola- } \\
\text { borativa nas equipes } \\
\text { da atenção primaria à } \\
\text { saúde. }\end{array}$ & $\begin{array}{l}\text { Previato, GF, } \\
\text { Baldissera, } \\
\text { VDA. } 2018 .\end{array}$ & $\begin{array}{c}\text { Analisar a Prática Interprofissional } \\
\text { Colaborativa em Saúde na perspectiva } \\
\text { de profissionais das equipes em atuação } \\
\text { na Atenção Primária à Saúde. }\end{array}$ & $\begin{array}{c}\text { Exploratório descri- } \\
\text { tivo com abordagem } \\
\text { qualitativa. }\end{array}$ \\
\hline 9 & SciELO & $\begin{array}{l}\text { Prática interprofis- } \\
\text { sional no serviço de } \\
\text { emergência: atribui- } \\
\text { ções especificas e } \\
\text { compartilhadas dos } \\
\text { enfermeiros. }\end{array}$ & $\begin{array}{l}\text { Batista REA, } \\
\text { Peduzzi M. } \\
2019 .\end{array}$ & $\begin{array}{l}\text { Mapear e categorizar, de acordo com a } \\
\text { Classificação das Intervenções de Enfer- } \\
\text { magem, as atribuições específicas dos } \\
\text { enfermeiros e identificar as comparti- } \\
\text { Ihadas com médicos e fisioterapeutas } \\
\text { nos Serviço de Emergência. }\end{array}$ & $\begin{array}{l}\text { Exploratório descri- } \\
\text { tivo de abordagem } \\
\text { qualitativa. }\end{array}$ \\
\hline 10 & MEDLINE & $\begin{array}{l}\text { A comunicação na } \\
\text { perspectiva dialógica } \\
\text { da prática interpro- } \\
\text { fissional colaborativa } \\
\text { em saúde na atenção } \\
\text { primária à saúde. }\end{array}$ & $\begin{array}{l}\text { Previato, GF, } \\
\text { Baldissera, } \\
\text { VDA. } 2018 .\end{array}$ & $\begin{array}{l}\text { Analisar a comunicação enquanto } \\
\text { domínio da prática interprofissional } \\
\text { colaborativa em Saúde no processo de } \\
\text { trabalho das equipes da Atenção Primá- } \\
\text { ria à Saúde. }\end{array}$ & $\begin{array}{c}\text { Descritivo interpreta- } \\
\text { tivo com abordagem } \\
\text { qualitativa. }\end{array}$ \\
\hline
\end{tabular}


As evidências na literatura mostraram que estudos sobre práticas interprofissionais em saúde e em enfermagem em UPA ainda são escassos, portanto, a análise das literaturas mostram ações interprofissionais que podem ser desenvolvidas, tais como, as reuniões, discussões de casos clínicos, planejamento de ações, educação permanente e uso de prontuário, utilizando a comunicação, colaboração interprofissional e trabalho em equipe. Além disso, o diagnóstico e as intervenções de enfermagem também são ações consideradas colaborativas nas práticas interprofissionais em saúde. Diante disso, entende-se que as práticas que podem ser desenvolvidas de modo interprofissional são de caráter educativo, gerencial e assistencial.

Estudo aponta que para a realização de um trabalho compartilhado a prática da reunião torna-se uma ação importante, pois, por meio dela é possível que os profissionais da saúde realizem discussões para propor ações que podem ter abordagens individuais ou coletivas. A partir de reuniões é possível desenvolver estratégias de atendimento e cuidado, bem como discutir casos clínicos ${ }^{14}$.

A prática interprofissional colaborativa em saúde possibilita alcançar o que o Sistema Único de Saúde preconiza em todas as suas instâncias de maneira efetiva, ou seja, a troca permanente de conhecimentos e experiências entre os profissionais, uma prática de colaboração e cooperação, bem como o aprendizado e interação entre diferentes profissões para melhorar a assistência aos usuários. Para alcançar essas práticas é importante a realização da educação permanente em saúde, facilitando conhecer o papel do outro na prática ${ }^{15}$.

Além disso, a literatura mostra que a educação permanente realizada para aproximar os profissionais da perspectiva interprofissional do trabalho em saúde é uma estratégia para tornar o trabalho mais colaborativo. Sendo assim, é importante que a instituição planeje mecanismos que sensibilizem os profissionais sobre a importância dessas práticas, do acolhimento, respeito a opinião do outro e planejamento das ações para que seja realizado com comunicação horizontal favorecendo troca de diálogo ${ }^{16}$.

Dessa forma, a sensibilização da gestão é essencial para o desenvolvimento de ações em saúde que sejam interprofissionais, tendo apoio de um assistente social, enfermeiro, coordenação entre outros ${ }^{17}$. A colaboração é muito importante na atenção ao paciente seja na rotina ou durante a emergência, desde um cuidado como corrigir a posição do paciente no leito, em que se faz necessário a ajuda do outro, ou até mesmo cuidados mais complexos como o apoio da equipe durante uma parada cardiorrespiratória para reverter às situações complexas no serviço de saúde de forma efetiva. Além disso, a troca de informação contribui para uma conduta terapêutica adequada. A sintonia entre a equipe permite um cuidado integrado e coeso, desde a decisão sobre isolamento de paciente até sobre as dificuldades de sair da ventilação mecânica, entre outros ${ }^{18}$.

Portanto, entende-se que os profissionais da saúde ao agirem de forma colaborativa e em equipe contribuem para a efetivação das práticas interprofissionais em saúde ${ }^{19}$. Nessa perspectiva o enfermeiro tem papel essencial, pois, desenvolve uma prática colaborativa. Realiza práticas autônomas por meio do processo de enfermagem, através do julgamento clínico terapêutico. Para efetivar as ações, é necessário um trabalho colaborativo da equipe, sendo que os enfermeiros participam na execução e monitoramento das intervenções ${ }^{20}$. Além disso, o enfermeiro é reconhecido por outros profissionais por sua prática colaborativa, sendo considerados referência e suporte para a equipe multiprofissional ${ }^{21}$.

Sendo assim, entende-se que as ações de saúde compartilhadas permitem uma abordagem integral e amplia a qualidade da atenção à saúde. O remodelamento das profissões da área da saúde que compõe a equipe de emergência representa a oportunidade de mudança do modelo de atenção na perspectiva interprofissional, colaborativa e centrada no paciente e na família, favorecendo a satisfação dos pacientes e profissionais e o uso adequado de recursos ${ }^{10}$.

\section{DISCUSSÃO}

A interprofissionalidade consiste na união de duas ou mais profissões atuando juntas, compartilhando saberes por um objetivo em comum, oferecer um cuidado com excelência ${ }^{12}$. Neste sentido podemos destacar o trabalho em equipe, que é a associação entre trabalho e interação de pessoas.

A educação interprofissional é parte fundamental e está presente em todas as áreas de conhecimento, em particular na saúde acontece quando dois ou mais profissionais inter-relaciona um com o outro, para melhorar a cooperação e a qualidade do cuidado ${ }^{22}$. A comunicação também é fundamental para a qualidade do atendimento, possibilitando a colaboração dos profissionais e a realização dos cuidados ao paciente ${ }^{3}$.

A colaboração interprofissional é uma estratégia do trabalho em equipe que consiste no processo de convívio entre diferentes profissões que praticam o processo de comunicação e tomadas de decisões compartilhadas na realização do cuidado 5 . Nessa perspectiva, é necessário que a formação em saúde seja direcionada a interprofissionalidade, que é uma estratégia que surgiu à medida que houve um declínio do modelo fragmentado de saúde ${ }^{23}$. Diante disso, o Ministério da Saúde implantou no ano de 2004, a Política Nacional de Educação Permanente em Saúde (PNEPS) como uma estratégia do SUS para formar e qualificar profissionais e colaboradores, unindo o ensino, os serviços e a comunidade. Abordando a metodologia da problematização e aprendizagem significativa, como perspectiva de renovação do $S U S^{24}$.

O mundo tem considerado a colaboração interprofissional como essencial para enfrentar os importantes impasses sociais e sanitários, pois aumenta a resolubilidade e obtém maior qualidade na assistência ${ }^{25}$. Neste sentido, a interprofissionalidade valoriza e expande saberes e habilidades, tendo como protagonista o usuário que interage com as diferentes práticas profissionais que atuam junto para cuidar melhor da saúde da população $0^{26}$.

Neste sentido, a educação interprofissional nas formações em saúde e seus efeitos são tão novos e requerem vivências próprias que vem necessitar de um empenho mais rigoroso e organizado, que possa fazer uma comparação nos resultados entre uma educação uniprofissional e interprofissional. E dentro desse contexto, avaliar as ações dos alunos com diferentes experiências de aprendizados no momento em que se formam, podendo ter uma contribuição e adesão no conhecimento de uma educação interprofissional ${ }^{23}$. 


\section{CONCLUSÃO}

A prática interprofissional em saúde e enfermagem é pouco explorada na área de urgência e emergência, ela requer o trabalho em equipe colaborativo dos diferentes profissionais. É notório a importância e necessidade de promover essa prática para que tenham um atendimento mais qualificado, satisfação do usuário e dos profissionais. Dessa forma, sugere-se que sejam desenvolvidas pesquisas de intervenção educativa a fim de fomentar essa prática nas Unidades de Pronto Atendimento.

\section{REFERÊNCIAS}

1. Ministério da Saúde (BR). Portaria n 10 de 3 de janeiro de 2017 Redefine as diretrizes de modelo assistencial e financiamento de UPA $24 \mathrm{~h}$ de Pronto Atendimento como Componente da Rede de Atenção às Urgências, no âmbito do Sistema Único de Saúde. 2017.

2. Silva PA, Silva GML, Rodrigues JD, Moura PV, Carminha IO, Ferreira DKS. Atuação em equipes multiprofissionais de saúde: uma revisão sistemática. ConScientiae Saúde, 2013;12(1):153-160.

3. Araújo TAM, Vasconcelos ACCP, Pessoa TRRF, Forte FDS. Multiprofissionalidade e interprofissionalidade em uma residência hospitalar: o olhar de residentes e preceptores. Interface (Botucatu). 2017; 21(62):601-13.

4. Peduzzi M, Oliveira MAC, Silva JAM, Agreli HLF, Neto MVM. Trabalho em equipe, prática e educação interprofissional. Clínica médica, Barueri: Manole; 2016.

5. Arruda, LS, Moreira COF. Colaboração interprofissional: um estudo de caso sobre os profissionais do Núcleo de Atenção ao Idoso da Universidade Estadual do Rio de Janeiro (NAI/UERJ), Brasil. Interface 2018; 22(64):199-210.

6. Peduzzi M, Agreli, HF. Trabalho em equipe e prática colaborativa na atenção primaria à saúde. Interface (Botucatu). 2018; 22(Supl. 2):1525-34.

7. Cunha ACM, Salomão AFS, Correia HPC, Yogui JOS. Educação interprofissional no cenário da atenção primaria a saúde: relato de experiencia. Rev. APS. 2017 abr/jun; 20(2): 306 - 306.

8. D'Amour D, Goulet L, Labadie JF, Rodriguez LSM, Pineault R. A model and typology of collaboration between professionals in healthcare organizations. BMC Health Services Research, London, 2008, v.8, n.188, p. 1- 14.

9. Freire Filho JR. Costa MV, Magnago C, Forster AC. Atitudes para a colaboração interprofissional de equipes da Atenção Primária participantes do Programa Mais Médicos. Rev. Latino-Am. Enfermagem 2018;26:e3018.

10. Batista REA, Peduzzi M. Prática Interprofissional colaborativa no serviço de emergência: atribuições privativas e compartilhadas dos fisioterapeutas. Interface (Botucatu). 2018; 22(Supl. 2):168595.

11. Batista REA, Peduzzi M. Prática interprofissional no Serviço de Emergência: atribuições específicas e compartilhadas dos enfermeiros. Rev Bras Enferm [Internet]. 2019;72(Suppl 1):228-9.

12. Costa MV, Peduzzi M, Freire Filho JR, Silva CBG. Educação interprofissional em saúde. Natal: SEDIS-UFRN, 2018.

13. Souza MT, Silva, MD, Carvalho R. Revisão integrativa: o que é e como fazer. einstein. 2010; 8(1 Pt 1):102-6.
14. Matuda CG, Pinto NRS, Martins CL, Frazão P. Colaboração interprofissional na estratégia saúde da família: implicações para a produção do cuidado e a gestão do trabalho. Ciência \& Saúde Coletiva, 20(8):2511-2521, 2015.

15. Previato GF, Baldissera VDA. Retratos da prática interprofissional colaborativa nas equipes da atenção primária à saúde. Rev Gaúcha Enferm. 2018;39:e2017-0132.

16. Farias DN, Ribeiro KSQS, Anjos UU, Brito GEG. Interdisciplinaridade e interprofissionalidade na estratégia saúde da família. Trab. Educ. Saúde, Rio de Janeiro, v. 16 n. 1, p. 141-162, jan./abr. 2018.

17. Reuter CLO, Santos VCF, Ramos AR. O exercício da interprofissionalidade e da intersetorialidade como arte de cuidar: inovações e desafios. Esc Anna Nery 2018;22 (4):e20170441.

18. Goulart BF, Parreira BDM, Noce LGA, Henriques SH, Simões ALA, Chaves LDP. Relacionamento interpessoal: identificação de comportamentos para trabalho em equipe em unidade coronariana. REME - Rev Min Enferm. 2019;23:e-1197.

19. Previato GF, Baldissera, VDA. A comunicação na perspectiva dialógica da prática interprofissional colaborativa em saúde na Atenção Primária à Saúde. Care. Interface (Botucatu). 2018; 22(Supl. 2):1535-47.

20. Santos GLA, Valadares GV, Santos SS, Moraes CRBM, Mello JCM, Vidal LLS. Prática colaborativa interprofissional e assistência em enfermagem. Escola Anna Nery 24(3)2020.

21. Caram CS, Rezende LC, Brito MJM. Prática colaborativa: potencialidades e desafios para o enfermeiro no contexto hospitalar. REME - Rev Min Enferm. 2017;21:e-1070.

22. Pereira MF. Interprofissionalidade e saúde: conexões e fronteiras em transformação. Interface. 2018; 22(Supl. 2):1753-6.

23. Toassi RFC. Interprofissionalidade e formação na saúde: onde estamos? organizadora. - 1.ed. - Porto Alegre: Rede UNIDA, 2017.

24. Brasil. Ministério da Saúde. Política Nacional de Educação Permanente em Saúde: o que se tem produzido para o seu fortalecimento?/, Secretaria de Gestão do Trabalho e da Educação na Saúde, Departamento de Gestão da Educação na Saúde - 1. ed. rev. - Brasília: Ministério da Saúde, 2017.

25. Matuda CG, Aguiar, DML, Frazão P. Cooperação interprofissional e a Reforma Sanitária no Brasil: implicações para o modelo de atenção à saúde. Saúde Soc. São Paulo, v.22, n.1, p.173-186, 2013.

26. Freire Filho, JR. Silva CBG, Costa MV, Forster AC. Educação Interprofissional nas políticas de reorientação da formação profissional em saúde no Brasil. Saúde debate. Rio de Janeiro, v. 43, n. spe1, p. 86-96, ago. 2019. 